\title{
Modulated magnetism in PrPtAl
}

\author{
Gino Abdul-Jabbar', Dmitry A. Sokolov', Christopher D. O'Neill', Christopher Stock', \\ Didier Wermeille ${ }^{2}$, Franz Demmel ${ }^{3}$, Frank Krüger ${ }^{3,4}$, Andrew G. Green ${ }^{4}$, \\ Florence Lévy-Bertrand ${ }^{5}$, Béatrice Grenier ${ }^{6}$ and Andrew D. Huxley ${ }^{1 \star}$
}

The transition between paramagnetism and ferromagnetism is the paradigm for a continuous phase transition at finite temperature. When such a transition is tuned to zero temperature in clean materials, the growth of low-energy zero-point fluctuations potentially drives an array of phenomena, including the formation of novel states such as nonconventional superconductivity. Experimentally, the growth of the fluctuations, however, is curtailed and the transition becomes discontinuous as its temperature is reduced. This is understood to arise from non-analytic corrections to the free energy that always occur ${ }^{1}$. In a recent theory ${ }^{2,3}$, changes of the excitation spectrum are self-consistently considered alongside the ground state. This analysis reveals that a transition to a new state may be an alternative outcome. As the excitation spectrum (the 'disorder') is pivotal to promoting the new 'order' this mechanism is referred to as 'order by disorder'. Here, we report the discovery of modulated order in PrPtAl, consistent with complex spirals, at the boundary between paramagnetism and ferromagnetism, giving the first clear experimental realization of such a state.

In our theoretical model, deformations of the Fermi surface in the modulated state enlarge the phase space available for low-energy particle-hole fluctuations and this self-consistently lowers the free energy relative to a uniform ferromagnetic state. Although previous theory predicting spiral formation ${ }^{4,5}$ based on this mechanism has considered isotropic magnets, easy-plane systems are better candidate materials, as a hard magnetic axis provides a natural orientation for the spiral wavevector and suppresses 'unwanted' moment fluctuations along the spiral direction. The anisotropy can be introduced with local moments ${ }^{6}$, although the theoretical description close to a ferromagnetic quantum critical point ${ }^{7}$ has only recently been extended to include the coupling of these moments to the conduction electrons ${ }^{8}$.

Here we describe our findings for PrPtAl. This material is close to being an easy-plane ferromagnet, but has an additional magnetic anisotropy between the two easy axes in the plane. The electronic levels of the praseodymium $4 \mathrm{f}^{2} \mathrm{Pr}^{3+}$ ions are split in the crystal environment (PrPtAl has an orthorhombic TiNiSn structure) into nine non-magnetic singlet states. Inelastic neutron studies ${ }^{9}$ reveal clear crystal field excitations between these. Choosing a system with only singlets simplifies the theory considerably, avoiding Kondo lattice physics, while still introducing magnetic anisotropy.

As there are no preformed moments, ferromagnetic order is achieved by mixing singlets via an inter-site exchange interaction ${ }^{10}$, a process referred to as induced-moment magnetism. Our theoretical approach to analysing the magnetic interactions that bring about magnetic order in PrPtAl differs from the standard treatment ${ }^{11}$ by keeping the full frequency dependence of the fermion-mediated Ruderman-Kittel-Kasuya-Yosida (RKKY) interaction. This is the key element for a description approaching a quantum critical point and is needed to capture the long-wavelength, low-energy behaviour of the free energy expressed as a functional of the local magnetization. The final result, apart from the inclusion of anisotropy, turns out to be similar to that for a fully itinerant system, but with the 'local' moments magnifying the magnetic response.

PrPtAl is reported in the literature to be a simple inducedmoment ferromagnet ${ }^{9,12}$ with a single transition from paramagnetism to ferromagnetism. Previous scattering studies ${ }^{9}$ correctly established that the Pr moments within the unit cell sum to give ferromagnetic order at low temperature (total moment directed along the a-axis), but with the four moments within a unit cell canted in opposing directions in the ac-plane. These measurements, however, did not look at temperatures close to the Curie temperature and previous macroscopic measurements were made in an applied magnetic field, which obscures the much richer underlying phase diagram that we report below.

Our measurements are on high-quality single crystals grown by the Czochralski technique (see Supplementary Information for sample preparation details). In zero magnetic field, we found that rather than a simple transition from paramagnetism (PM) to ferromagnetism (FM) there are three consecutive transitions. With decreasing temperature the first is at $T_{1}=5.85 \pm 0.05 \mathrm{~K}$ to a doubly modulated incommensurate spin density wave state (SDW1), followed by a second transition in the temperature range $5.7-5.3 \mathrm{~K}$ (centred at $T_{2}=5.5 \pm 0.1 \mathrm{~K}$ ) to a single incommensurate modulation (SDW2) of different period with a strong third-order harmonic. The third transition is to uniform ferromagnetism over the temperature interval $5.0-4.3 \mathrm{~K}$ (centred at $T_{\mathrm{FM}}=4.7 \pm 0.1 \mathrm{~K}$ ). This is seen in all four samples we have studied with neutron scattering (at D23, ILL and SPINS, NIST) and with resonant $\mathrm{X}$-ray scattering (at BM28, ESRF). Data obtained with resonant $\mathrm{X}$-ray scattering have the highest $q$-resolution and are shown in Fig. 1. The incommensurate diffraction signal is visible for an $\mathrm{X}$-ray energy at the $\operatorname{Pr} \mathrm{L}_{2}$ resonance edge $(6.444 \mathrm{keV})$, which fluorescence and absorbance measurements suggest is a simple dipole transition (see Supplementary Information for further experimental details). The sensitivity to the $\mathrm{X}$-ray incident energy and the observed intensity for neutron scattering at the same wavevector transfer prove that the satellites are of magnetic origin. In both SDW states the modulation vectors are precisely along the c-axis.

\footnotetext{
${ }^{1}$ School of Physics and CSEC, University of Edinburgh, Edinburgh EH9 3FD, UK. ${ }^{2}$ XMAS, ESRF, BP220, F-38043 Grenoble, France. ${ }^{3}$ ISIS, STFC, Rutherford Appleton Laboratory, Chilton, Didcot OX11 OQX, UK. ${ }^{4}$ London Centre for Nanotechnology, University College London, Gordon St., London WC1H OAH, UK. ${ }^{5}$ CNRS, Institut Néel, Grenoble 38042 Cedex 9, France. ${ }^{6}$ Université Grenoble Alpes \& CEA, INAC-SPSMS, F-38000 Grenoble, France. *e-mail: a.huxley@ed.ac.uk
} 


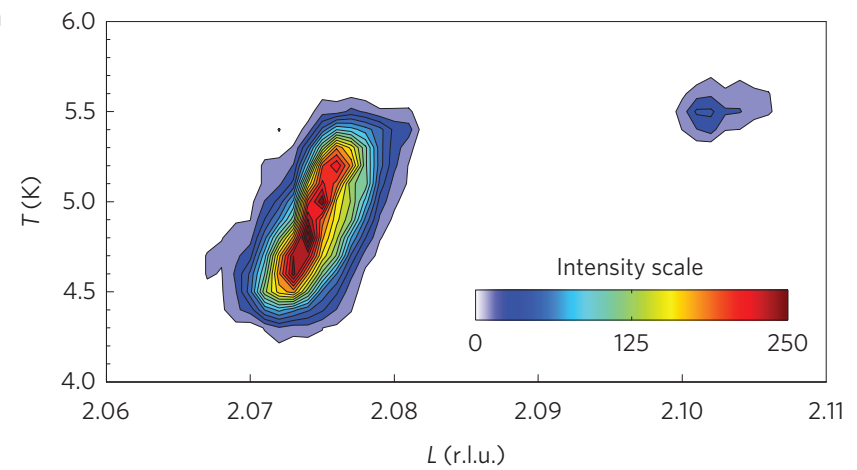

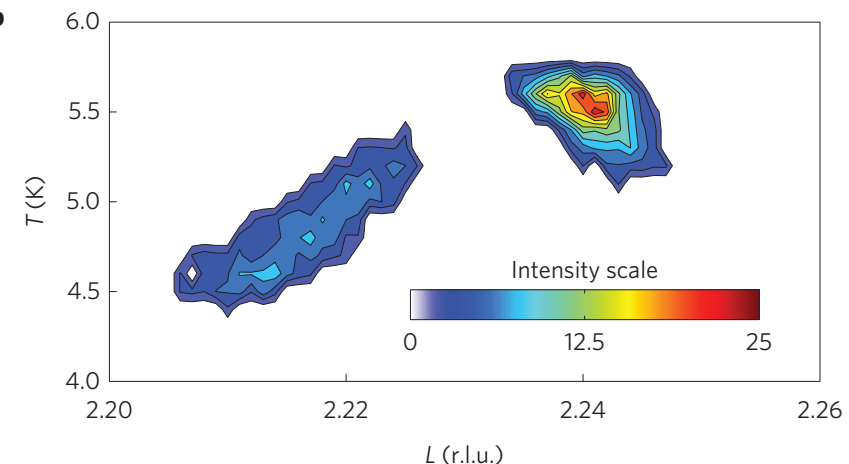

C

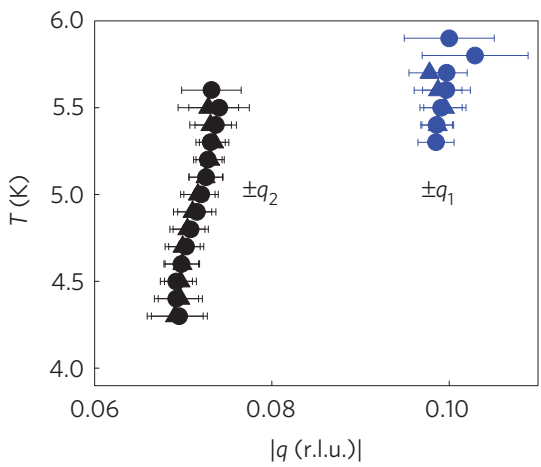

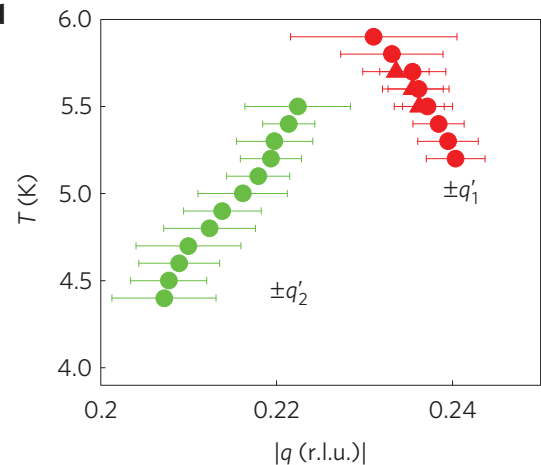

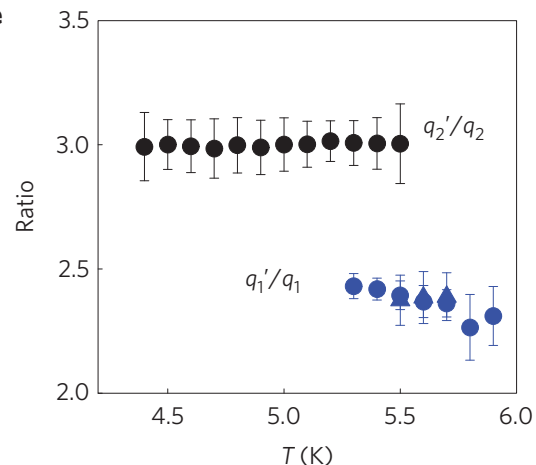

Figure 1 | Ordering wavevectors. a,b, Colour-scale images of diffracted intensity as a function of reciprocal coordinate $(0,0, L)$ and temperature for

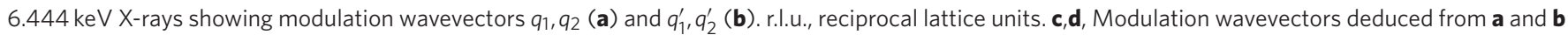

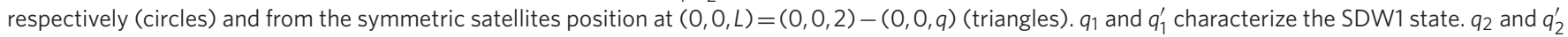
characterize the SDW2 state. $\mathbf{e}$, Ratio of the modulation vectors in each state.

To further explore the nature of the magnetic order we measured the spin dependence of the neutron cross-section at wavevector transfers $(0,0,2 \pm q)$ with the SPINS instrument at NIST. The incoming neutron-spin polarization was aligned with the crystal a-axis (which is the direction of the low-temperature ferromagnetic moment). In the SDW 2 state ( $q \approx 0.07$ reciprocal lattice units) we observed that the spin-flip (SF) scattering had an intensity 0.1 times that of the non-spin-flip (NSF) scattering. This implies that the modulated state has magnetic moments along the $\mathbf{b}$-axis as well as along the a-axis, suggesting that the SDW2 states could be an elliptical spiral.

The examination of the SF/NSF ratio for satellites close to other Bragg positions revealed that there is also a moment component along the c-axis (Supplementary Information). A magnetic structure for the SDW2 state consistent with these measurements is shown in Fig. 2. In the figure the moments rotate in a plane whose normal is inclined from the propagation direction. In addition there is an intra-cell antiferromagnetic moment (equivalent to that in the FM state), which for clarity is not shown. The maximum magnitude of the root mean square of the modulated moment perpendicular to the $\mathbf{c}$-axis is $0.72(5) \mu_{\mathrm{B}}$, compared with a low-temperature FM moment 0.95(6) $\mu_{\mathrm{B}}$ (ref. 9).

The presence of third-order harmonics provides further indirect evidence for spiral order in the SDW2 state. The moment direction for a simple spiral propagating along the $\mathbf{z}$-direction with moments turning in the xy-plane in the presence of crystalline anisotropy can be written as

$$
\frac{1}{|m|}\left(\begin{array}{l}
m_{x} \\
m_{y}
\end{array}\right)=\left(\begin{array}{c}
\cos \left[q_{\mathrm{m}} z-\phi(z)\right] \\
\sin \left[q_{\mathrm{m}} z-\phi(z)\right]
\end{array}\right)
$$

with $q_{\mathrm{m}}$ the principal modulation wavevector. The phase $\phi(z)$ allows the pitch of the spiral to vary with position to reduce the anisotropy energy. In this expression $\phi(z)$ is periodic with period $\pi / q_{\mathrm{m}}$, resulting in only odd harmonic components of the amplitude of the magnetization, with wavevector $q=(2 n+1) q_{\mathrm{m}}$ (for integer $n)$. For example, in a weak ellipsoidal (two-fold) anisotropy, the free energy is minimized for $\phi(z)=\delta \sin \left(2 q_{\mathrm{m}} z\right)$ ( $\delta$ is a constant determined by the strength of the anisotropy), resulting in a thirdorder amplitude $\delta / 2$. A strong four-fold anisotropy is required to explain the experimentally measured SF and NSF third-harmonic intensities for PrPtAl.

The changes of magnetic structure seen with diffraction also have signatures in thermodynamic measurements that we now describe, starting with the heat capacity. The heat capacity divided by temperature $C / T$ has cusp-like features at all three transitions (Fig. 3). The scatter in values at $T_{\mathrm{FM}}$ and $T_{2}$ depend on the temperature history, indicating that these transitions are almost certainly first order. $C / T$ extrapolates to a large value of around $40 \mathrm{~mJ} \mathrm{~mole} \mathrm{e}^{-1} \mathrm{~K}^{-2}$ at low temperature. This is consistent with a large electronic effective mass, which could arise from a strong interaction between conduction electrons and local moments. A strong localmoment conduction-electron interaction supports the order-bydisorder mechanism for modulated state formation described later.

The d.c. susceptibility - namely, the magnetization divided by the applied magnetic field $(M / H)$-and a.c. susceptibility $(\chi)$ are also shown in Fig. 3. The SDW2 state is clearly much less strongly polarizable than both the SDW1 and FM states. The distinct signatures of the modulated state in magnetization measurements are suppressed by a field, being washed out in a field of only $10 \mathrm{mT}$ (this explains why the modulated states were missed in earlier studies). An out-of-phase component of the a.c.-susceptibility $\chi^{\prime \prime}$ develops only below the lowest transition, with a corresponding reduction of the in-phase susceptibility $\chi^{\prime}$ relative to $M / H$. This marks the onset of dissipation (hysteresis) due to the formation of ferromagnetic domains. 

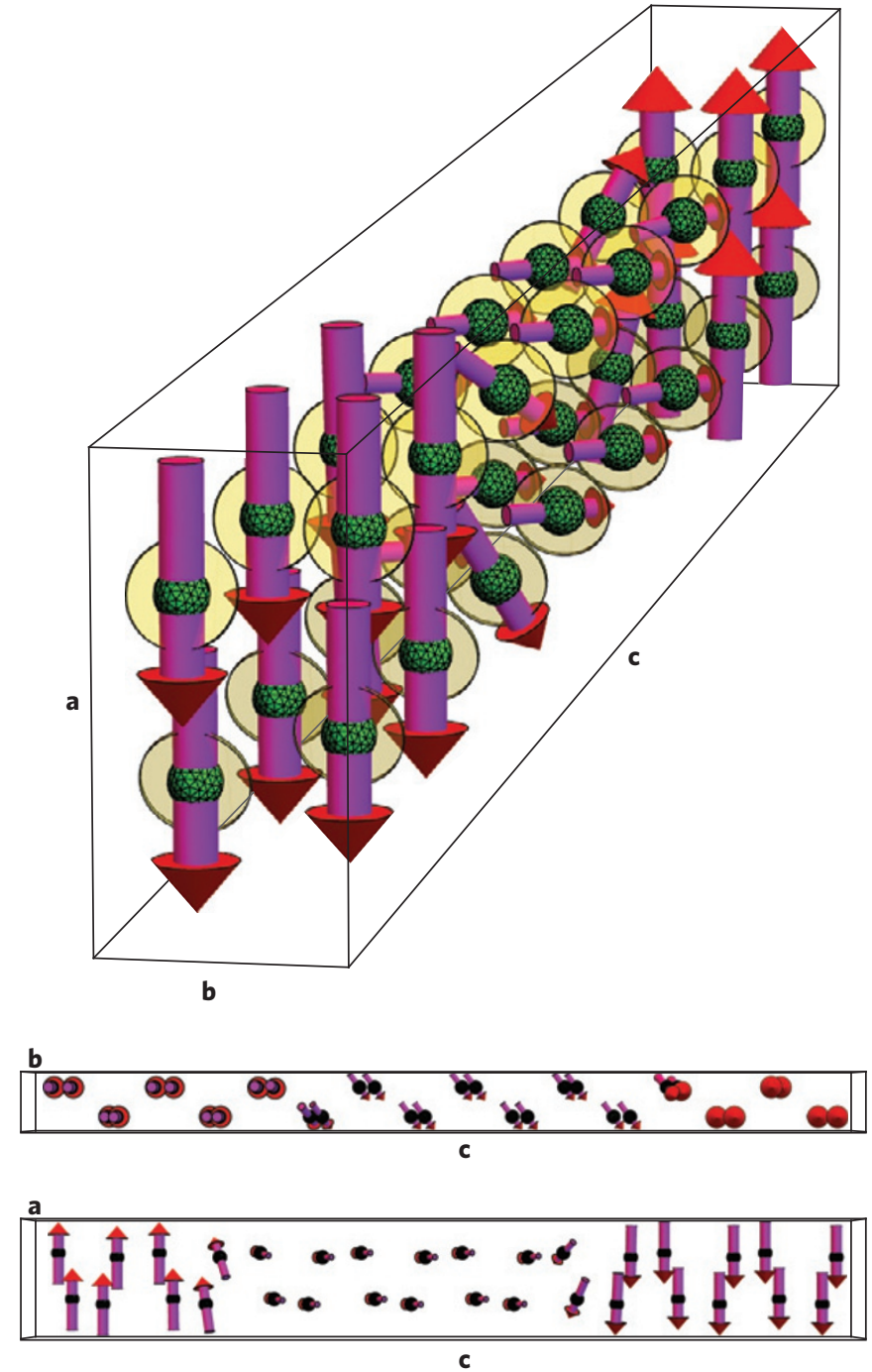

Figure 2 | The magnetic structure of the SDW2 state consistent with the reported diffraction studies. The upper image shows the SDW2 state over a length of approximately $1 / 2$ the modulation period. Only the $\operatorname{Pr}$ atoms are shown (spheres). These form chains running along the $\mathrm{c}$-axis with different a-coordinate, zigzagging in the bc-plane. The moments are shown as arrows. There is an additional intra-cell canting that tilts the moments out of the plane of the shaded discs in the c-direction (in the opposite sense in the two chains), which for clarity is not shown. The lower two images show the same structure viewed from the $\mathbf{a}$ and $\mathbf{b}$ crystal directions.

We now examine possible explanations for the formation of the modulated states, considering first two mechanisms that can be described in terms of local-moment physics alone.

The first mechanism applies when there is no inversion symmetry; the resulting Dzyaloshinskii-Moriya interaction then favours spiral magnetic order ${ }^{13}$, as found in $\mathrm{MnSi}$ (ref. 14). For $\mathrm{PrPtAl}$ there are crystal inversion symmetries linking Pr sites; therefore, there is no such interaction between them.

The second mechanism arises from competing near-neighbour exchange interactions, which can be tuned to give a Devil's staircase of large-period commensurate ordering vectors ${ }^{15}$, describing periodic patterns of domain walls. The theory has been suggested to apply to holmium metal ${ }^{16}$, where the moments rotate from one hexagonal plane of the structure to the next with a quasi-continuous evolution of $q$ with decreasing temperature, before $q$ locks to a fixed value at lower temperature. The absence of dissipation in

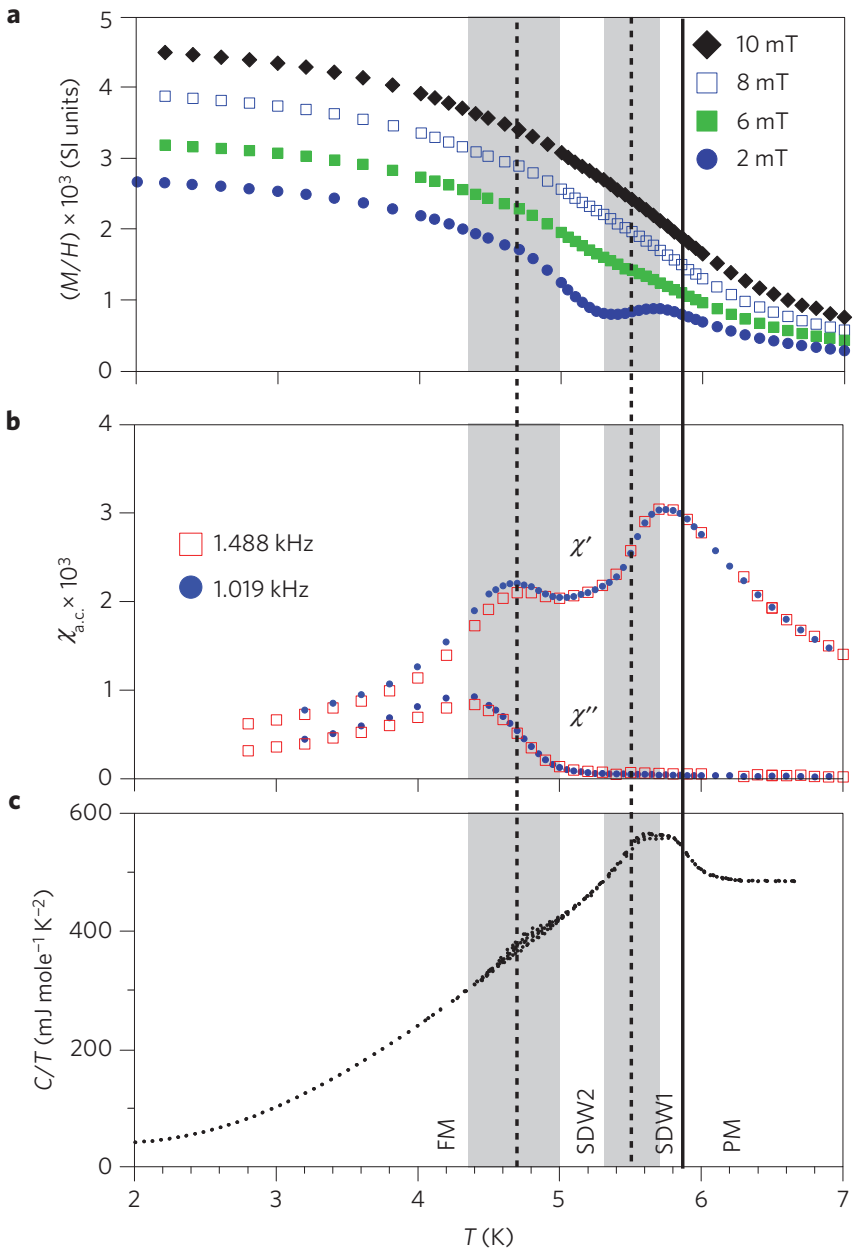

Figure 3 | Thermodynamic measurements for PrPtAl. a, The d.c. susceptibility (magnetization/applied magnetic field) for a field applied along the easy a-axis. $\mathbf{b}$, In-phase $\left(\chi^{\prime}\right)$ and out-of-phase $\left(\chi^{\prime \prime}\right)$ parts of the a.c. susceptibility measured in zero magnetic field at frequencies of 1.019 and $1.488 \mathrm{kHz}$. c, Heat capacity divided by temperature. The vertical lines passing through all panels show the transition temperatures seen with neutron and X-ray scattering, with the regions where multiple states coexist shaded.

the a.c. susceptibility in the SDW2 and SDW1 states and our observation that neutron-spin depolarization is found only below $T_{\mathrm{FM}}$ (Supplementary Information) rule out explanations based on domain walls for the modulated structures for PrPtAl. In PrPtAl $q$ also evolves continuously.

We now discuss explanations for the modulated states that depend more explicitly on the conduction electrons. Our observations for PrPtAl invite comparison with hexagonal $\mathrm{Tb}$ and Dy. Both Tb and Dy have modulated magnetic states below an ordering temperature $T_{N}$ and undergo first-order transitions at lower temperatures to uniform ferromagnetism ${ }^{17}$. We briefly describe the various theories that have been put forward for $\mathrm{Tb}$ and Dy and how the magnetism of PrPtAl is different.

The theories for $\mathrm{Tb}$ and Dy consider a long-range exchange interaction $J(q)$ between magnetic ions transmitted by the conduction electrons (known as the RKKY interaction) that is peaked at an initial ordering vector. As the temperature is lowered below $T_{N}$ the effect of magnetic anisotropy grows as a strong power of the ordered moment ${ }^{18}$, leading to a reduction of the ordering wavevector and then a transition to ferromagnetism. The peak in $J(q)$ has been attributed to either a nesting of the 
Fermi surface ${ }^{19}$ or a Kohn anomaly ${ }^{20}$. In both cases the magnetic super-zone cell in the modulated state reduces the electronic density of states and contributes to lowering the energy in mean field theory. The theories have been reasonably successful in explaining the qualitative temperature evolution $q(T)$. Similar behaviour to $\mathrm{Tb}$ and $\mathrm{Dy}$ is also reported in some samples of $\mathrm{UCu}_{2} \mathrm{Si}_{2}$ (ref. 21); in this case Kondo physics may be involved as well as Fermi-surface nesting ${ }^{22}$.

Substantial Fermi-surface nesting for a 3D material is extremely rare. One example is found in $\alpha$-uranium ${ }^{23}$, where it gives rise to a charge density wave, rather than to a spin density wave. For the rare-earth elements, including $\mathrm{Tb}$ and $\mathrm{Dy}$, a webbing feature at the zone boundary specific to their hexagonal crystal structure may provide such a special case ${ }^{19}$. The band structure for PrPtAl is not known, but that for LaPtAl (ref. 24) provides a guide, considering the $\operatorname{Pr} f$-electrons to be localized. For LaPtAl there are no apparent nesting vectors along the c-direction. Nesting is therefore unlikely to underlie modulated state formation in PrPtAl.

For the SDW1 state $q_{1}^{\prime}$-increases with decreasing temperature, which is the opposite trend from that in the above materials. We focus our discussion on the SDW2 state, which spans a wider temperature interval and for which the temperature dependence and magnitude of $q_{2}(T)$ is similar to the $q(T)$ behaviour encountered in the rare earths. However, there are important differences in other quantities. For both Dy (ref. 25) and Tb (ref. 26) there is a marked increase of electrical resistance entering the modulated state when the electrical current is parallel to the modulation vector. This is explained by the formation of a super-zone cell, which gaps the Fermi surface and therefore reduces the density of states. For PrPtAl, in contrast, the resistivity falls with decreasing temperature (Fig. 4d) for currents along all the crystal axes. There is a small hysteresis of up to $1 \%$ in the resistance in the ordered state below $T_{1}$; the value in increasing temperature is lower than in decreasing temperature, confirming that states PM, SDW1, SDW2 and FM have successively lower resistivity. Another difference from the rare-earth elements concerns the behaviour in a magnetic field applied along the easy axis (the a-axis), which we describe next.

For hexagonal $\mathrm{Tb}$ and Dy there is a transition from a helix to a fan state and then to ferromagnetism with magnetic field. The field at which the upper transition occurs depends on the temperature, but has a maximum value near $T_{N}$ of around $1 \mathrm{~T}$ for Dy (ref. 27) and $0.5 \mathrm{~T}$ for $\mathrm{Tb}$ (ref. 28). For the above theory the transition field is expected to be of the order of $(J(0)-J(q)) / \mathcal{M}$ (ref. 29), in agreement with the observed fields ( $\mathcal{M}$ is the magnetic moment). For PrPtAl the energy dispersion of the lowest crystal field excitation gives an energy difference $E(0)-E\left(q_{1,2}\right) \approx 0.2 \mathrm{meV}$ (Supplementary Information), which corresponds to a field in excess of $2 \mathrm{~T}$. We find a much lower field is needed to suppress the modulated state, as described below.

Figure 4a shows the magnetoresistance (MR) at different temperatures. The magnetoresistance is observed to be negative in the FM, SDW2 and PM states, including at low field. This contrasts with the behaviour seen in the modulated states of the rare-earth elements, where an initial positive magnetoresistance is observed and attributed to the formation of higher-order super-zone gaps ${ }^{30}$. The behaviour we observe is, however, consistent with the orderby-disorder model, which predicts an increase in the density of states in the modulated state that is suppressed with field. The observed negative magnetoresistance indicates that this more than compensates any contribution to the resistance from field-induced higher-order super-zone gaps in SDW2.

For the SDW1 state the initial magnetoresistance is positive, passing through a peak at around $10 \mathrm{mT}$ before becoming negative at higher magnetic field. The field needed to suppress a similar feature in the rare-earth elements corresponds to
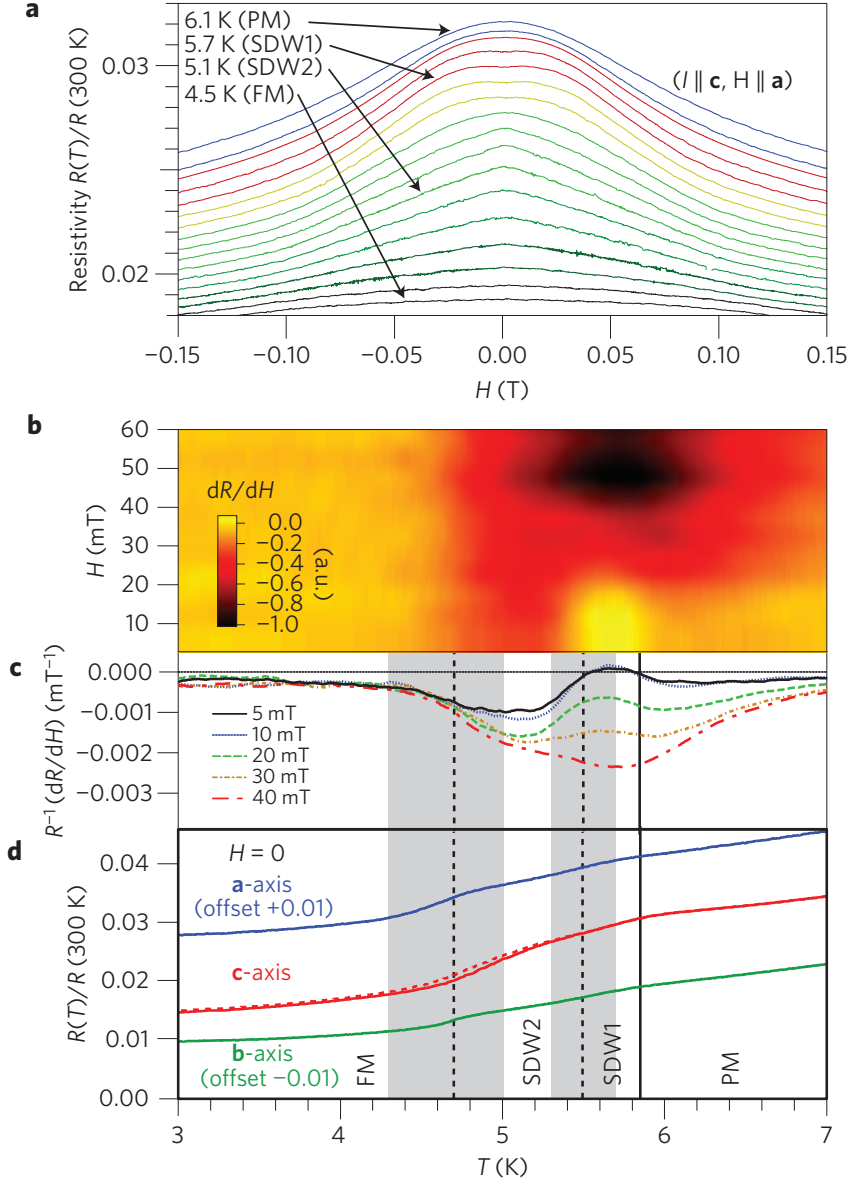

Figure 4 | Electrical resistivity of PrPtAl. a, Resistance (normalized to its zero-field value at $300 \mathrm{~K}$ ) as a function of magnetic field for different temperatures in the range 4.5 to $6.1 \mathrm{~K}$ in steps of $0.1 \mathrm{~K}$ (the magnetic field is parallel to the a-axis $(H \| \mathbf{a})$ and the current is parallel to the $\mathbf{c}$-axis $(I \| \mathbf{c})$ ). An initial positive magnetoresistance is observed only in the SDW1 state. b. Colour plot of the field-cooled differential magnetoresistance (decreasing the temperature from the paramagnetic state at each field). c, Differential magnetoresistance resistance at several magnetic fields. d, Zero-field resistance relative to the value at $300 \mathrm{~K}$ as a function of temperature for different current directions. The solid lines are for increasing temperature, the dashed line is for decreasing temperature for the $\mathbf{c}$-axis. The vertical solid and dashed lines in $\mathbf{c}, \mathbf{d}$ indicate the transition temperatures between the different magnetic states PM, SDW1, SDW2 and FM, with the regions of coexistence shaded (as determined from the $\mathrm{X}$-ray and neutron scattering measurements).

the field that suppresses the modulated $\operatorname{state}^{30}$. The differential magnetoresistance at different fields and temperatures is shown in Fig. 4 b,c. These figures show that there is no remnant of the positive magnetoresistance feature in fields above $40 \mathrm{mT}$. This suggests that the SDW1 structure is suppressed by a modest field of less than $40 \mathrm{mT}$. The suppression of the features in the magnetization below $10 \mathrm{mT}$ (Fig. 3a) has already been commented on. These fields are several orders of magnitude below $2 \mathrm{~T}$. A different description from that used for the modulated states in the rareearth elements is therefore required to explain both SDW1 and SDW2 states.

Below we show that non-analyticities of the free energy approaching quantum criticality can explain our findings of extreme sensitivity to magnetic field and account for a fall in resistivity on entering the modulated states. Modulated state formation is predicted on general grounds when the Curie temperature is small. 
a

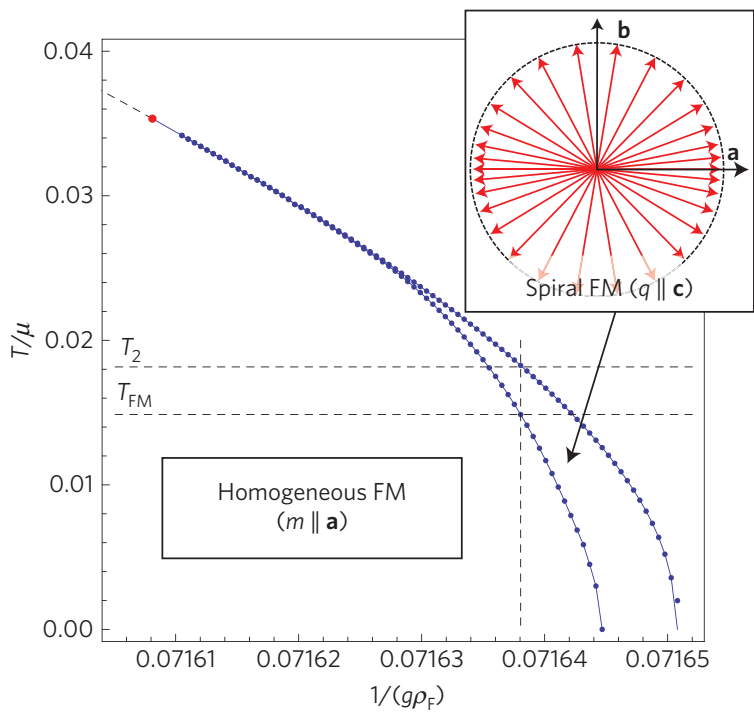

c

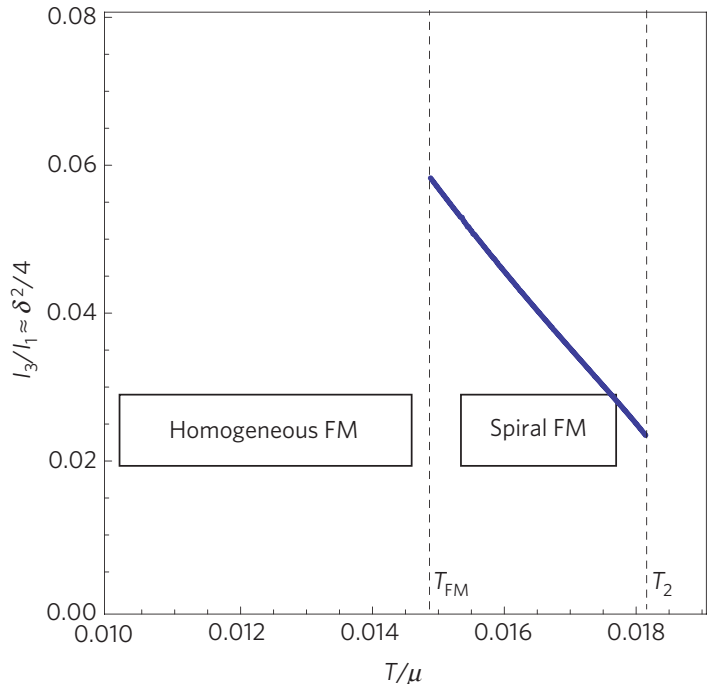

b

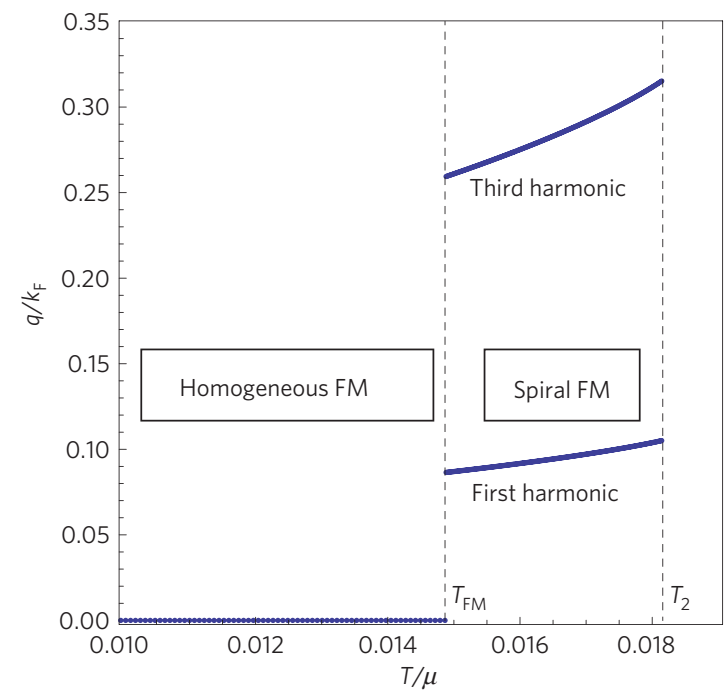

d

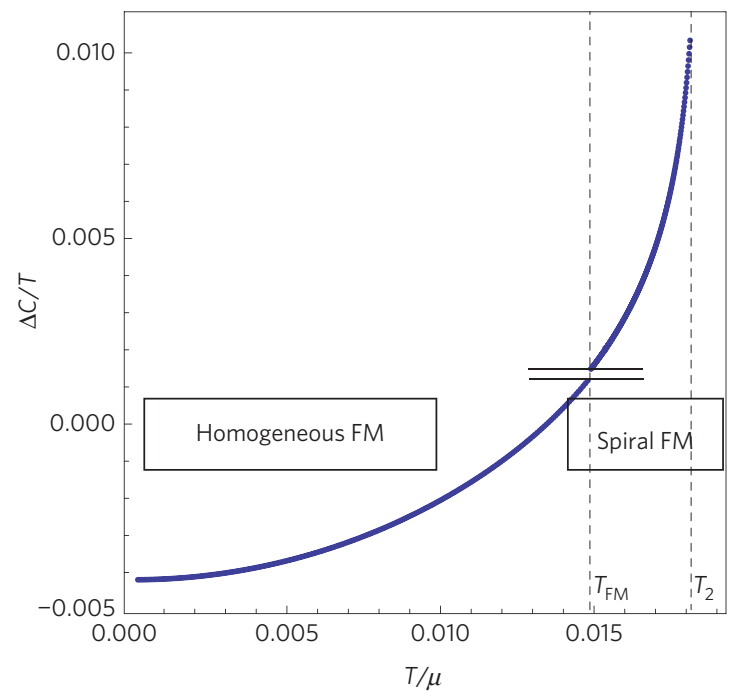

Figure $\mathbf{5}$ | Theoretical predictions of the quantum order-by-disorder model. a, Phase diagram as a function of temperature $T$ and inverse electron interaction strength $g$ ( $\rho_{\mathrm{F}}$ is proportional to the density of states at the Fermi energy). The vertical dashed line shows a value of $g$ that would give transitions with increasing temperature from ferromagnetism to a (spiral) modulated state and then to paramagnetism. The inset shows the dominant anisotropy-induced deformation of the spiral. $\mathbf{b}$, Evolution of the spiral ordering wavevector $q$ (in units of the Fermi wavevector $\mathrm{k}_{\mathrm{F}}$ ) as a function of temperature. $\mathbf{c}$, Intensity ratio $I_{3} / h_{1}$ of the third and first harmonics in the spiral phase. $\mathbf{d}$, Magnetic contribution to the heat capacity, $\Delta C / T$ (in units $k_{F}^{3} / \mu$ ).

Moreover, the ordering vector does not have to match any special feature of the Fermi-surface geometry.

The long-wavelength model consists of conduction electrons at chemical potential $\mu$, interacting via a local repulsion $g$ and coupled to the local moments $\mathbf{J}$ of the $\operatorname{Pr}^{3+}$ ions with coupling constant $\gamma$. The Hamiltonian is

$$
\begin{gathered}
H=\int_{\mathbf{r}}\left\{\left(\psi_{\uparrow}^{\dagger}, \psi_{\downarrow}^{\dagger}\right)\left[-\nabla^{2}-\mu-\gamma \sum_{\alpha} J_{\alpha}(\mathbf{r}) \sigma_{\alpha}\right]\left(\begin{array}{l}
\psi_{\uparrow} \\
\psi_{\downarrow}
\end{array}\right)\right. \\
\left.+g \psi_{\uparrow}^{\dagger} \psi_{\uparrow} \psi_{\downarrow}^{\dagger} \psi_{\downarrow}+\frac{1}{2} \sum_{\alpha} \chi_{\alpha}^{-1} J_{\alpha}^{2}(\mathbf{r})\right\}
\end{gathered}
$$

where $\sigma_{\alpha}(\alpha=x, y, z)$ denote Pauli matrices and $\psi_{\nu}(\mathbf{r})(\nu=\downarrow, \uparrow)$ are electronic field operators. The electron dispersion is taken to be isotropic. Magnetic anisotropy is induced through the van Vleck susceptibility of the local moments $\left(\chi_{a}>\chi_{b} \gg \chi_{c}\right)$. In the temperature range where the spiral forms, the local susceptibility has a small temperature dependence, which we neglect in the following. For sufficiently strong interactions $g$, the model exhibits a FM ground state with a moment along the a-axis.

The central idea of the quantum order-by-disorder approach is to self-consistently compute fluctuations and their contribution to the free energy near the quantum critical point for different magnetically ordered states. This reproduces a non-analytic freeenergy contribution $\Delta f_{0} \sim m^{4} \ln \left(m^{2}+T^{2}\right)$ for a homogeneous FM state that leads to a first-order transition at low temperatures. The first-order transition is, however, pre-empted by the formation of an incommensurate spiral state. The free energy for modulated states can be obtained from the expression for the uniform state by noting that the electron dispersion becomes $\epsilon_{ \pm}(\mathbf{k})=k^{2} \pm \sqrt{\left(k_{z} q\right)^{2}+(g m)^{2}}$ in the presence of spiral order. The free-energy density is then $f(m, q)=f_{0}(m)+f_{2}(m) q^{2}+f_{4}(m) q^{4} / 2$, where the functions $f_{2}$ and $f_{4}$ can be deduced from the homogeneous result $f_{0}$ (Supplementary Information).

Including the local moments in the above analysis leads to the same free-energy expression plus an additional anisotropy 
term $f_{\text {anis }}(\mathbf{r})=\sum_{\alpha} u_{\alpha} m_{\alpha}^{2}(\mathbf{r})$ with $u_{\alpha}=-2 \gamma^{2} /\left(\chi_{\alpha}^{-1}+2 \gamma^{2} / g\right)$. In this expression $\mathbf{m}$ is the total moment, which is composed of a conduction electron and local-moment contribution, $\mathbf{m}=\mathbf{m}_{\text {cond }}+(\gamma / g) \mathbf{J}$. The local moment is given by $J_{\alpha}=\left(2 \gamma /\left(\chi_{\alpha}^{-1}+2 \gamma^{2} / g\right)\right) m_{\alpha}$.

As for the fully itinerant theory, the finite range of the interaction and the effect of weak disorder can be included by introducing two further parameters whose values determine the tri-critical temperature and extent of the modulated region (see Supplementary Information). The resulting phase diagram is shown in Fig. 5a. Over a range of electron interactions $g$, we find a sequence of transitions on cooling from a paramagnet to a modulated spiral $(q \| \mathbf{c})$ and then to $\mathrm{FM}(m \| \mathbf{a})$.

A key prediction of the quantum order-by-disorder model is the increase of the spiral ordering wavevector with temperature (Fig. 5b). This behaviour is indeed observed in the modulated phase SDW2. The anisotropy in the ab-plane renders the FM/spiral transition first order with a small change in magnetic moment but a relatively big jump of $q$.

The anisotropy also causes the deformation $\phi(z)=\delta \sin (2 q z)$ of the spiral (see Fig. 5b) as described earlier, giving rise to odd higher harmonics in the magnetic structure factor. As shown in Fig. $5 c$, we find typical values of $5 \%$ for the intensity ratio $I_{3} / I_{1}=\delta^{2} / 4$ between third and first harmonics. The spectral weight of the third harmonic is further enhanced by modulations of the moment magnitude, $m[1+\tilde{\delta} \cos (2 q z)]$, corresponding to an elliptical deformation of the spiral. With increasing anisotropy between $\mathbf{a}$ - and $\mathbf{b}$-directions, the deformations of the spiral increase slightly up to the point where the spiral phase is destroyed. A more complete description could include crystal field parameters (at present not known) and quantum spin states for the local moment to better account for the actual anisotropy as a function of localmoment direction.

The calculated magnetic contribution to the specific heat is shown in Fig. 5d. The Sommerfeld coefficient $\Delta C / T$ increases monotonically and shows a sharp drop at the transition to the paramagnet state. The FM-spiral transition is characterized by a small jump, as well as a latent heat that could explain the scatter in the values of the experimental heat capacity close to $T_{\mathrm{FM}}$. Although the latent heat divided by the temperature at $T_{\mathrm{FM}}$ is small (around $5 \%$ of the jump in $\Delta C$ at $T_{2}$ ) the transition is sufficiently first order to give a positive step in $\Delta C / T$ with increasing temperature (in contrast with a negative step for a weakly first-order or secondorder phase transition). A higher density of states (and higher heat capacity) in the modulated state compared with the FM state is a general signature of the order-by-disorder phenomena. A small positive step in the experimental heat capacity may be inferred by extrapolating the heat capacity from either side of the SDW2-FM transition, although the scatter in the data close to the transition makes an accurate comparison difficult. The density of states in the modulated state is also increased by a factor $\left[1+1 / 8\left(q / k_{\mathrm{F}}\right)^{2}\right]$ relative to the $\mathrm{PM}$ state. Both the residual electrical resistivity and the rate of increase of the resistivity with temperature are sensitive to the density of states. An increased density of states decreases the residual resistivity while increasing electron-electron scattering, which can give a stronger temperature dependence. This accounts well for the changes in the electrical resistivity seen experimentally (Fig. 4d).

The energy stabilizing the spiral formation relative to ferromagnetism is small, meaning that the spirals are very sensitive to an applied magnetic field along the easy axis. For the above model parameters, a magnetic field of only a few millitesla is sufficient to suppress the modulated state (Supplementary Information).

The modulated state is also predicted to be sensitive to sample quality and would be absent in lower-quality samples with shorter mean-free path (Supplementary Information). The heat capacity reported in the literature ${ }^{31}$ for arc-melted polycrystals does not show any signature of the modulated states supporting this conclusion.

If quantum criticality indeed underlies the modulated state formation, the range of temperature over which the spiral state exists relative to the ferromagnetic state should increase as the Curie temperature is reduced. We have made measurements at different pressures that indeed show this to be the case (Supplementary Information). The transition temperatures rise with pressure and become closer together, which corresponds to an increase of conduction-electron interaction, $g$.

Given the simplicity of the above calculation, its success in accounting for the observed phenomena in PrPtAl is remarkable. A more quantitative comparison requires more detailed knowledge of the band structure and crystalline electric field than at present available. Consideration of multiple bands might then also afford an explanation for the doubly modulated state SDW1.

The induced-moment magnet PrPtAl thus provides the first example of modulated state formation at the border between ferromagnetism and paramagnetism, driven by quantum criticality. The phenomena we have observed agree with many of the predictions for quantum critical theory. This validates an interesting alternative to transitions becoming discontinuous approaching the ferromagnetic-paramagnetic QCP. It avoids cutting off the divergence of critical fluctuations, although the divergence is shifted to finite wavevectors. Such fluctuations are not linked to a Fermisurface nesting vector and represent a new type of quantum critical behaviour. The changed nature of the fluctuations may possibly lead to different quantum ordered states emerging at lower temperatures and be relevant to the formation of unconventional superconductivity. In particular, even when a modulated state does not form, soft fluctuations associated with incipient modulated state formation, centred at small but finite values of $q$, may provide the glue for magnetic pairing.

\section{Received 3 September 2014; accepted 29 December 2014;} published online 9 February 2015

\section{References}

1. Belitz, D., Kirkpatrick, T. R. \& Vojta, T. Nonanalytic behavior of the spin susceptibility in clean Fermi systems. Phys. Rev. B 55, 9452-9462 (1997).

2. Chubukov, A. V., Pépin, C. \& Rech, J. Instability of the quantum-critical point of itinerant ferromagnets. Phys. Rev. Lett. 92, 147003 (2004).

3. Rech, J., Pépin, C. \& Chubukov, A. V. Quantum critical behavior in itinerant electron systems: Eliashberg theory and instability of a ferromagnetic quantum critical point. Phys. Rev. B 74, 195126 (2006).

4. Conduit, G. J., Green, A. G. \& Simons, B. D. Inhomogeneous phase formation on the border of itinerant ferromagnetism. Phys. Rev. Lett. 103, 207201 (2009).

5. Karahasanovic, U., Kruger, F. \& Green, A. G. Quantum order-by-disorder driven phase reconstruction in the vicinity of ferromagnetic quantum critical points. Phys. Rev. B 85, 165111 (2012).

6. Roussev, R. \& Millis, A. J. Quantum critical effects on transition temperature of magnetically mediated $p$-wave superconductivity. Phys. Rev. B 61, 140504R (2001).

7. Belitz, D., Kirkpatrick, T. R. \& Vojta, T. First order transitions and multicritical points in weak itinerant ferromagnets. Phys. Rev. Lett. 82, 4707-4710 (1999)

8. Kirkpatrick, T. R. \& Belitz, D. Universal low-temperature tricritical point in metallic ferromagnets and ferrimagnets. Phys. Rev. B 85, 134451 (2012).

9. Kitazawa, H. et al. Magnetic structures of the rare-earth platinum aluminides RPtAl ( $\mathrm{R}=\mathrm{Ce}, \mathrm{Pr}, \mathrm{Nd})$. J. Solid State Chem. 140, 233-241 (1998).

10. Bleaney, B. Crystal field effects and the co-operative state. I. A primitive theory. Proc. R. Soc. Lond. A 276, 19-27 (1963).

11. Jensen, J. \& Macintosh, A. R. Rare Earth Magnetism, Structures and Excitations (Clarendon, 1991).

12. Hullinger, F. On new ternary aluminides LnPdAl and LnPtAl. J. Alloys Compd. 196, 225-228 (1993)

13. Bak, P. \& Jensen, M. H. Theory of helical magnetic structures and phase transitions in MnSi and FeGe. Physica C 13, L881-L885 (1980).

14. Pappas, C. et al. Chiral paramagnetic skyrmion-like phase in MnSi. Phys. Rev. Lett. 102, 197202 (2009). 
15. Bak, P. E. \& von Boehm, J. Ising model with solitons, phasons, and "the devil's staircase". Phys. Rev. B 21, 5297-5308 (1980).

16. Cowley, R. A. \& Bates, S. The magnetic structure of holmium. I. J. Phys. C 21 , 4113-4124 (1988).

17. Dietrich, O. W. \& Als-Nielsen, J. Neutron diffraction study of the magnetic long-range order in Tb. Phys. Rev. B 162, 315-320 (1967).

18. Miwa, H. \& Yosida, K. Magnetic ordering in the rare-earth metals with more than half-filled $4 f$ shells. Prog. Theor. Phys. 26, 693-721 (1961).

19. Keeton, S. C. \& Loucks, T. L. Electronic structure of rare-earth metals. I. Relativistic augmented-plane-wave calculations. Phys. Rev. 168, 672-678 (1968).

20. Miwa, H. Variation of the period of the magnetic ordering in the heavy rare earth metals. Proc. Phys. Soc. 85, 1197-1207 (1965).

21. Honda, F., Metoki, N., Matsuda, T. D., Haga, Y. \& Onuki, Y. Long-period, longitudinal spin density modulation in an itinerant $5 \mathrm{f}$ magnetic compound $\mathrm{UCu}_{2} \mathrm{Si}_{2}$. J. Phys. Condens. Matter 18, 479-492 (2006).

22. Tróc, R., Samsel-Czekala, M., Stepień-Damm, J. \& Coqblin, B. Interplay between ferromagnetism, SDW order, and underscreened Kondo lattice in $\mathrm{UCu}_{2} \mathrm{Si}_{2}$. Phys. Rev. B 85, 224434 (2012).

23. Fast, L. et al. Theoretical aspects of the charge density wave in uranium. Phys. Rev. Lett. 81, 2978-2981 (1998).

24. Ueda, T. et al. de Haas-van Alphen effect and the electronic state in CePtAl. J. Magn. Magn. Mater. 310, 391-392 (2007).

25. Wilding, M. D. \& Lee, E. W. Superzone boundary effects in the electrical resistivity of dysprosium. Proc. Phys. Soc. 85, 955-961 (1965).

26. Hegland, D. E., Legvold, S. \& Spedding, F. H. Magnetization and electrical resistivity of terbium single crystals. Phys. Rev. 131, 158-162 (1963).

27. Herz, R. \& Kronmüller, H. Field-induced magnetic phase transitions in dysprosium. J. Magn. Magn. Mater. 9, 273-275 (1978).
28. Zverev, V. I. et al. Magnetic and magnetothermal properties and the magnetic phase diagram of high purity single crystalline terbium along the easy magnetization direction. J. Phys. Condens. Matter 26, 066001 (2014).

29. Kitano, Y. \& Nagamiya, T. Magnetisation process of a screw spin system II. Prog. Theor. Phys. 31, 1-43 (1964).

30. Benito, L., Ward, R. C. C. \& Blamire, M. G. Positive magnetoresistance induced by fan-type phases in a spin-spiral magnet. Phys. Rev. B 88, 224407 (2013).

31. Kato, S., Kitazawa, H. \& Kido, G. Magnetic properties of $\operatorname{Pr}_{1-x} \operatorname{La}_{x} \mathrm{PtAl}$ Physica B 281-282, 128-129 (2000).

\section{Acknowledgements}

Support from the Royal Society (A.D.H.), EPSRC (A.G.G., A.D.H., C.D.O’N., D.A.S., F.K.) and SUPA (C.S.) is gratefully acknowledged.

\section{Author contributions}

F.L-B., G.A-J. and D.A.S grew the single-crystal samples. G.A-J., C.D.O'N. and D.A.S. performed the macroscopic measurements. Central facility measurements were made by G.A-J., F.L-B., D.A.S., D.W., F.D., B.G. and C.S. F.K. performed the theoretical calculations. The theoretical work was overseen by A.G.G. and the experimental work by A.D.H. F.K. and A.D.H. wrote the manuscript. All authors discussed the data and contributed to the analysis.

\section{Additional information}

Supplementary information is available in the online version of the paper. Reprints and permissions information is available online at www.nature.com/reprints. Correspondence and requests for materials should be addressed to A.D.H.

\section{Competing financial interests}

The authors declare no competing financial interests. 